\title{
Thrombosis in an Internal Jugular Vein and an Upper Limb Deep Vein Treated with Edoxaban
}

\author{
Mizuho Toratani, Akiko Hayashi, Naoki Nishiyama, Hidehiko Nakamura, Ryuji Chida, \\ Takaaki Komatsu, Shiro Nakahara, Sayuki Kobayashi and Isao Taguchi
}

\begin{abstract}
A 45-year-old man complained of swelling of the left side of his neck and left upper limb. Ultrasonography and enhanced computed tomography (CT) revealed thrombosis of the left internal jugular, subclavian, and brachiocephalic vein. Based on various examinations, the patient was diagnosed with idiopathic venous thrombosis early in his clinical course. There were no findings to suggest malignancy or abnormal coagulability. However, two months after the start of treatment, the patient was diagnosed with gastric cancer. Despite the presence of Trousseau syndrome, treatment with edoxaban (an oral anticoagulant), reduced the swelling dramatically without any bleeding complications.
\end{abstract}

Key words: upper-extremity deep vein thrombosis, Trousseau syndrome, oral anticoagulant

(Intern Med 56: 1053-1055, 2017)

(DOI: 10.2169/internalmedicine.56.7405)

\section{Case Report}

A 45-year-old man presented to our department with a 7day history of swelling of the left side of his neck and left upper limb. Ultrasonography and enhanced computed tomography (CT) revealed thrombosis of the left internal jugular vein, left subclavian vein, and left brachiocephalic vein (Fig. 1A and B, 2). The results of chest radiography and electrocardiography were normal. Laboratory examinations found an elevated white blood cell count $(11,000 / \mu \mathrm{L})$, and C reactive protein $(2.65 \mathrm{mg} / \mathrm{dL})$, D-dimer $(11.62 \mu \mathrm{g} / \mathrm{mL})$, fibrin degradation product $(13.3 \mu \mathrm{g} / \mathrm{mL})$, and fibrinogen (>700 mg/dL) levels. The levels of protein S, protein $\mathrm{C}$, and antithrombin III were normal, and a test for antiphospholipid antibody syndrome was negative. The other findings on ultrasonography and enhanced CT were unremarkable; however, a ventilation-perfusion scan revealed a pulmonary thromboembolism. The patient's tumor marker levels, including carcinoembryonic antigen $(1.9 \mathrm{ng} / \mathrm{mL})$, carbohydrate antigen 19-9 (32.9 U/mL), alpha fetoprotein $(4.2 \mathrm{ng} / \mathrm{mL})$, and prostate specific antigen $(0.662 \mathrm{ng} / \mathrm{mL})$ were within normal limits, and there was no sign of melena. The patient was diagnosed with idiopathic venous thrombosis. His vital signs were stable, and he underwent treatment with unfractionated heparin and edoxaban (an oral anticoagulant). Treatment with unfractionated heparin (10,000 units/day, continuous drip infusion) for 7 days did not affect the patient's swelling; however, his D-dimer level was reduced to 7.01 $\mu \mathrm{g} / \mathrm{mL}$. The activated partial thromboplastin time during the heparin treatment had been between 46 seconds and 57 seconds, which was the therapeutic range. In addition, edoxaban $(60 \mathrm{mg} / \mathrm{day}$, orally) was administered at the same time that heparin was stopped. After 7 days of edoxaban treatment, the swellings of the patient's left neck and left upper limb were smaller, and his D-dimer had decreased to 3.87 $\mu \mathrm{g} / \mathrm{mL}$. One month later, ultrasonography showed smaller thrombi and increased blood flow through the left subclavian and brachiocephalic veins (Fig. 1C). For 1 month during these treatments, the patient's condition (including his symptoms, vital signs and appetite) improved and there were no bleeding complications. At two months after the start of therapy, chest radiography revealed the rapid onset of bilateral pleural effusion. A cytological examination of the pleural effusion revealed adenocarcinoma, and endoscopy identified gastric cancer. The histopathological examination of a biopsy specimen found poorly differentiated adenocarcinoma and signet ring cell carcinoma of the stomach. The present 

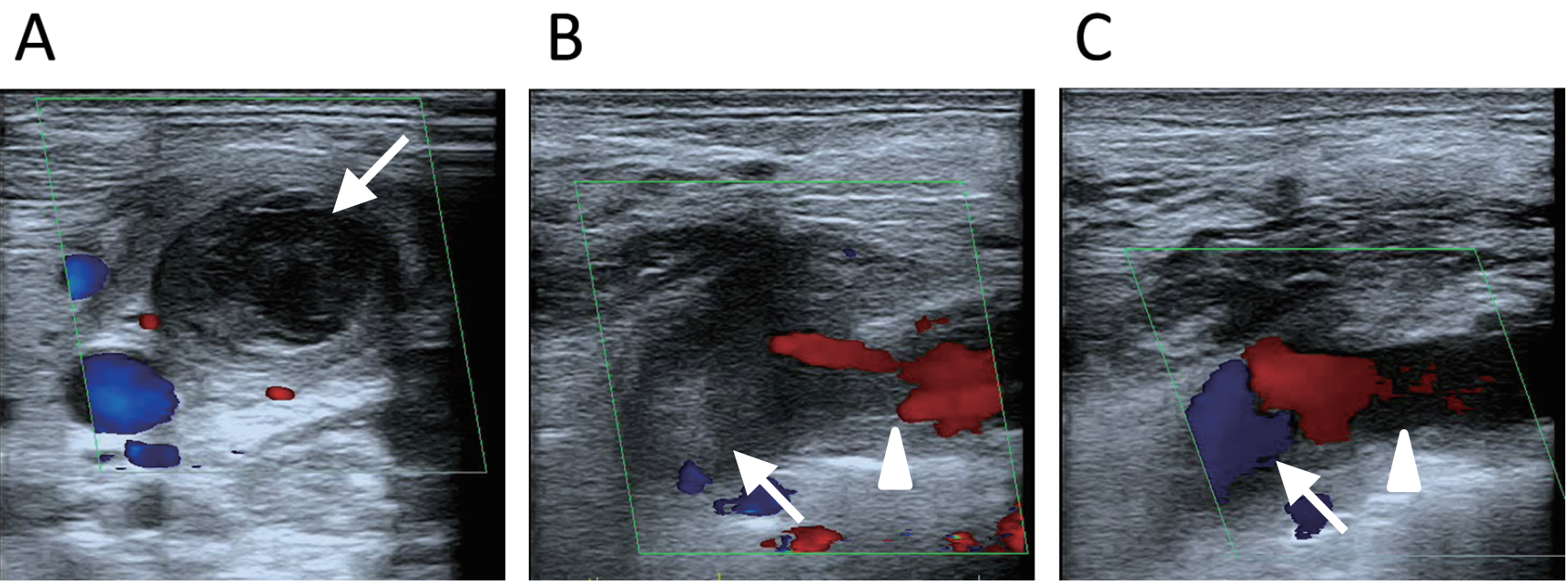

Figure 1. Ultrasonography images (color flow Doppler imaging). A: A short-axis view of the left side of the neck. On the first ultrasound examination, a hyperechoic region of the thrombus in the left jugular vein thrombus coexisted with a hypoechoic region, which occluded the left jugular vein (arrow). B: The left subclavian approach. On the first ultrasound, a thrombus was present in the left subclavian vein (triangle) and another thrombus was present in the left brachiocephalic vein (arrow). C: The left subclavian approach. After 1 month of edoxaban treatment, the thrombi in the left subclavian vein (triangle) and left brachiocephalic vein (arrow) were smaller, and blood flow through these veins was increased.

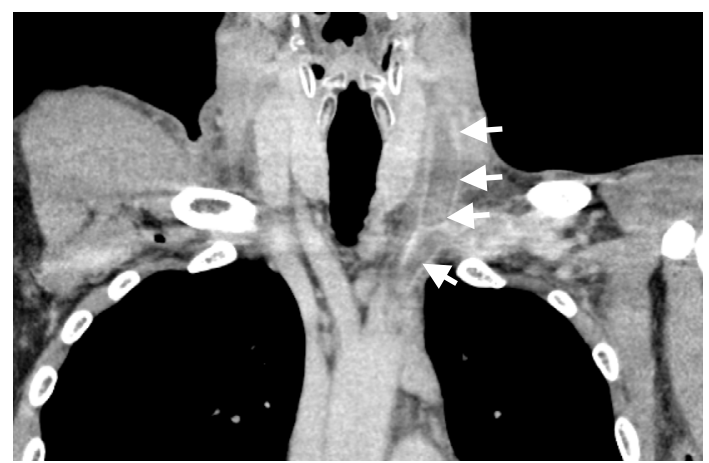

Figure 2. Contrast-enhanced computed tomography showing thrombi in the left internal jugular vein, left subclavian vein, and left brachiocephalic vein (arrows).

case was thus diagnosed with malignancy-related thromboembolism or Trousseau syndrome. Gastric cancer treatment was initiated.

\section{Discussion}

The prevalence of upper-extremity deep vein thrombosis (UEDVT), which includes thrombosis of the axillary, subclavian, or brachiocephalic veins, ranges from $1-4 \%$ of all cases of DVT. Primary UEDVT (20\% of all UEDVT) occurs due to activity-related venous trauma. Secondary UEDVT (80\% of all UEDVT) is almost always due to central venous catheters and malignancy (1). Malignancy-related thromboembolism is also known as Trousseau syndrome (2). Our patient had jugular vein thrombosis and left UEDVT, which were associated with malignancy.
UEDVT is rare; however, it is associated with high rates morbidity and mortality (29-40\%) because of the risk of pulmonary embolism (PE), post-thrombotic syndrome, and the loss of vascular access. The prevalence of UEDVT in patients with central venous catheters ranges from 27 to $66 \%$ (3). Lindbland et al. reported that $63 \%$ of patients with a known UEDVT had Trousseau syndrome (4). The rate of PE due to secondary UEDVT ranges from 15 to $25 \%$, with an autopsy-proven rate of up to $50 \%$ (3). We found several reports on internal jugular vein thrombosis associated with malignancy (lung cancer, prostate cancer, malignant lymphoma, ovarian cancer, and uterine cancer) (5-9).

Our patient was first diagnosed with idiopathic venous thrombosis, because he was otherwise asymptomatic, had no other CT findings, and because his occupation involved the installation of air conditioners, which required the abduction of his upper limbs. Additionally, all of the tumor markers were within the normal ranges, and he did not report melena. However, when we encounter UEDVT in a patient without a central venous catheter, we must investigate the possible presence of malignancies. If ultrasonography and CT are unsuccessful in revealing the cause of UEDVT, other diagnostic modalities should be used, including endoscopy.

The patient's venous thrombosis, which was ultimately found to be associated with gastric cancer, was effectively treated using edoxaban. Ultrasonography clearly demonstrated decreases in the size of the thrombi and increased blood flow through the veins. Ultrasonography is noninvasive, and does not require nephrotoxic contrast or ionizing radiation. Furthermore, because the diagnostic accuracy of ultrasonography is good (sensitivity $78-100 \%$, specificity 
$82-100 \%$ ), it is suitable for the diagnosis and follow up of UEDVT (1).

The administration of standard anticoagulation therapy to patients with DVT traditionally includes an initial period of treatment in which either unfractionated heparin or lowmolecular-weight heparin are administered for 5-7 days, followed by warfarin for at least 3 months (1). In our case, edoxaban was effective for reducing the malignancy-related venous thrombi. The Hokusai-venous thromboembolism (VTE) study, which included patients with cancer, demonstrated that edoxaban was non-inferior to high-quality standard therapy and was associated with significantly less bleeding in a broad spectrum of patients (10). The HokusaiVTE-cancer study is now being conducted to evaluate whether edoxaban is non-inferior to low-molecular-weight heparin for the treatment of VTE in patients with cancer (11). Thus, the anticoagulation therapy for patients with malignancy-related venous thrombosis may change.

It is difficult to maintain a therapeutic international normalized ratio (INR) in patients with cancer. These patients are often at risk of drug interactions, and warfarin bioavailability is unpredictable in cancer patients, who may have vomiting, malnutrition, or diarrhea. The clinical decisions regarding the optimum mode of antithrombotic therapy for cancer patients with DVT will be impacted by the risk of bleeding, the perioperative period, and the mode of administration (namely, by oral, sub-cutaneous injection or intravenous injection). Thus, it is essential to develop an individualized approach to anticoagulation therapy for the patients with malignancy-related DVT.

Eoxaban therapy may be non-inferior to high-quality warfarin therapy and may cause significantly less bleeding. In addition, it is not necessary to measure or control the INR with edoxaban. Furthermore, it is not necessary to administer edoxaban and heparin simultaneously, because edoxaban can rapidly exert its pharmacological action after oral intake. In the present case, although there was no period in which both edoxaban and heparin were administered, the swelling of the left side of the patient's neck and left upper limb improved after 7 days of edoxaban treatment with no bleeding complications, despite the presence of gastric cancer.

The authors state that they have no Conflict of Interest (COI).

\section{References}

1. Sajid MS, Ahmed N, Desai M, Baker D, Hamilton G. Upper limb deep vein thrombosis: a literature review to streamline the protocol for management. Acta Haematol 118: 10-18, 2007.

2. Varki A. Trousseau's syndrome: multiple definitions and multiple mechanisms. Blood 110: 1723-1727, 2007.

3. Verso M, Agnelli G. Venous thromboembolism associated with long term use of central venous catheters in cancer patients. J Clin Oncol 19: 3665-3675, 2003.

4. Lindbland B, Tengborn L, Bergqvist D. Deep vein thrombosis of the axillary and subclavian veins: epidemiological data, effects of different types of treatment and late sequelae. Eur J Vasc Surg 2: 161-165, 1988.

5. Shameem M, Akhtar J, Bhargava R, Ahmed Z, Baneen U, Khan NA. Internal jugular vein thrombosis: a rare presentation of mediastinal lymphoma. Respir Med CME 3: 273-275, 2010.

6. Erkoç R, Uzun K, Yuca K, et al. Internal juglar vein thrombosis two different etiologies. Eur J Med 2: 123-128, 2005.

7. Kunimasa K, Korogi Y, Okamoto Y, Ishida T. Spontaneous internal jugular vein thrombosis associated with lung cancer. Intern Med 52: 1849, 2013.

8. Togashi Y, Kim YH, Masago K, et al. Pulmonary embolism due to internal jugular vein thrombosis in a patient with non-small cell lung cancer receiving bevacizumab. Int J Clin Oncol 16: 444-446, 2011.

9. Naganuma T, Dote K, Kato M, et al. Pulmonary embolism due to internal jugular vein thrombosis without an indwelling catheter. Intern Med 48: 433-436, 2009.

10. Büller HR, Décousus H, Grosso MA, et al. Edoxaban versus warfarin for the treatment of symptomatic venous thromboembolism. N Engl J Med 369: 1406-1415, 2013.

11. van Es N, Di Nisio M, Bleker SM, et al. Edoxaban for treatment of venous thromboembolism in patients with cancer. Rationale and design of the Hokusai VTE-cancer study. Thromb Haemost 114: 1268-1276, 2015.

The Internal Medicine is an Open Access article distributed under the Creative Commons Attribution-NonCommercial-NoDerivatives 4.0 International License. To view the details of this license, please visit (https://creativecommons.org/licenses/ by-nc-nd/4.0/).

(C) 2017 The Japanese Society of Internal Medicine http://www.naika.or.jp/imonline/index.html 